\title{
Time-resolved photoelectron spectroscopy of wavepackets through a conical intersection in $\mathrm{NO}_{2}$
}

\author{
Yasuki Arasaki, ${ }^{1}$ Kazuo Takatsuka, ${ }^{1, a)}$ Kwanghsi Wang, ${ }^{2}$ and Vincent McKoy ${ }^{2}$ \\ ${ }^{1}$ Department of Basic Science, Graduate School of Arts and Sciences, The University of Tokyo, \\ Komaba, 153-8902 Tokyo, Japan \\ ${ }^{2}$ Laboratory for Molecular Sciences, California Institute of Technology, \\ Pasadena, California 91125, USA
}

(Received 15 January 2010; accepted 2 March 2010; published online 30 March 2010)

\begin{abstract}
We report the results of theoretical studies of the time-resolved femtosecond photoelectron spectroscopy of quantum wavepackets through the conical intersection between the first two ${ }^{2} A^{\prime}$ states of $\mathrm{NO}_{2}$. The Hamiltonian explicitly includes the pump-pulse interaction, the nonadiabatic coupling due to the conical intersection between the neutral states, and the probe interaction between the neutral states and discretized photoelectron continua. Geometry- and energy-dependent photoionization matrix elements are explicitly incorporated in these studies. Photoelectron angular distributions are seen to provide a clearer picture of the ionization channels and underlying wavepacket dynamics around the conical intersection than energy-resolved spectra. Time-resolved photoelectron velocity map images are also presented. () 2010 American Institute of Physics.
\end{abstract}

[doi:10.1063/1.3369647]

\section{INTRODUCTION}

Femtosecond time-resolved photoelectron spectroscopy is a versatile probe of ultrafast dynamics in molecules and has been applied in recent years to a variety of systems and processes. ${ }^{1-5}$ It is particularly well suited to the study of wavepacket dynamics in nonadiabatic systems where the nuclear and electronic modes are coupled. Time-resolved photoelectron spectra probe the entire configuration space spanned by the evolving wavepacket and hence, in principle, can provide information along all energetically accessible internuclear geometries. Where the electronic-nuclear mixing cannot be readily discerned from a photoelectron energy analysis alone, photoelectron angular distributions should in general still reflect changes in the character of electronic states during a nonadiabatic process.

Conical intersections (CIs) are ubiquitous in polyatomic molecules and are among the most important of nonadiabatic processes in these systems. They play a fundamental role in the excited-state dynamics of simple polyatomic molecules and are also believed to be responsible for the underlying photostability of DNA under ultraviolet radiation. ${ }^{6-8}$ Femtosecond time-resolved photoelectron spectroscopy can be expected to be a versatile probe of wavepacket dynamics in and around CIs. In fact, internal conversion in polyatomic molecules was among the earliest suggested application of this technique, ${ }^{9}$ which was subsequently realized experimentally. ${ }^{10}$ Recently, Bisgaard et al. ${ }^{11}$ and Horio et $a l .{ }^{12}$ could also track the change in electronic character with vibrational motion in an excited state from measured molecular-frame and laboratory-frame photoelectron angular distributions, respectively. Advances in ultrashort pulse shap-

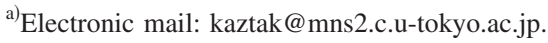

ing technology may also well enable the observation of wavepacket dynamics through a CI on the actual time scale of the nonadiabatic transition.

The CI between the first two ${ }^{2} A^{\prime}$ states of the $\mathrm{NO}_{2}$ molecule is known to lead to an extremely complex absorption spectra ${ }^{13-15}$ and has been the subject of numerous studies of nonadiabatic dynamics. ${ }^{16-22}$ For $C_{2 v}$ geometries the two surfaces $\left({ }^{2} A_{1}\right.$ and $\left.{ }^{2} B_{2}\right)$ intersect at a bond angle that depends on the bond length and form a one-dimensional CI seam. ${ }^{17}$ The seam is located close to the bottom of the excited state and is readily accessible by a vibrational wavepacket launched onto the excited electronic surface from the Franck-Condon region of the ground state. In a previous paper ${ }^{23}$ we investigated the application of femtosecond pump-probe photoelectron spectroscopy to explore the ultrafast dynamics near this $\mathrm{CI}$ in $\mathrm{NO}_{2}$ and showed that the energy-resolved photoelectron spectra could identify the passage of wavepackets through the CI. These studies, however, employed constant photoionization matrix elements and accounted only for ionization into the triplet cation. There ${ }^{23}$ we also noted the need to incorporate the geometry and energy dependences of the photoionization matrix elements and to include ionization into the singlet ground state of the cation in order to achieve a more realistic and useful description of the time-resolved photoelectron spectra of these wavepackets.

In this paper we extend these earlier studies by employing robust values of the photoionization matrix elements in calculations of the time-resolved photoelectron spectra for ionization into the singlet and triplet ion states. We also report the photoelectron angular distributions for these timeresolved spectra. We have previously demonstrated the utility of such time-resolved photoelectron angular distributions and the need to incorporate geometry- and energy-dependent photoionization matrix elements in studies of time-resolved photoelectron spectra in a series of papers tracking funda- 

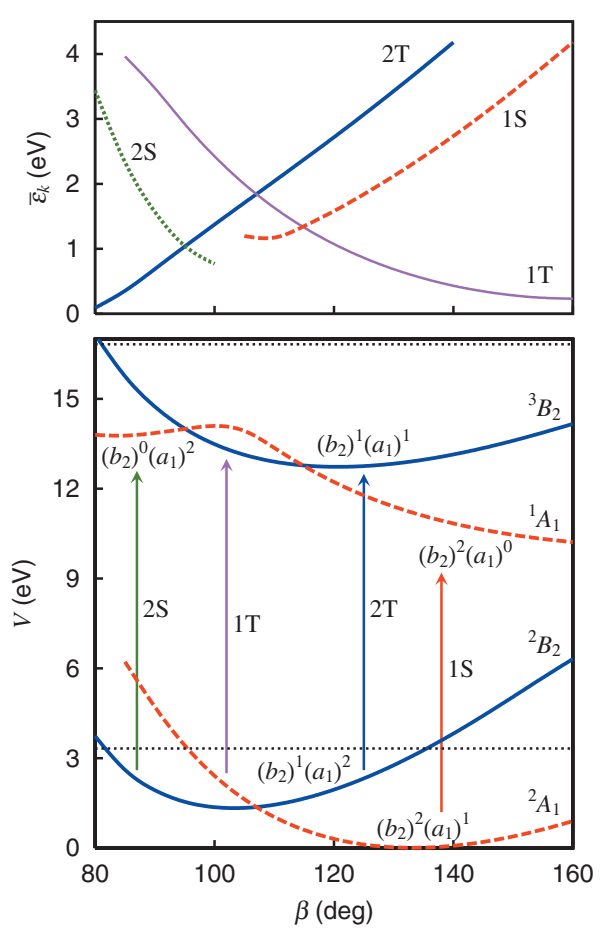

FIG. 1. Scheme to observe vibrational wavepacket dynamics through the ${ }^{2} A_{1} /{ }^{2} B_{2} \mathrm{CI}$ in $\mathrm{NO}_{2}$. The lower panel shows the two neutral surfaces ${ }^{2} A_{1}$ and ${ }^{2} B_{2}$ in the diabatic representation $\left(r_{1}=r_{2}=1.22 \AA, C_{2 v}\right.$ geometry $)$ and adiabatic representations of the two ion surfaces ${ }^{1} A_{1}$ and ${ }^{3} B_{2}$ used in these studies. The four possible ionization channels between these ion surfaces and the neutral states are indicated with arrows. The dominant configurations are shown along each potential curve. Dotted horizontal lines indicate the energy reached by the pump $\left(v_{0}+\omega_{\mathrm{pu}}=3.3\right)$ and probe pulses $\left(v_{0}+\omega_{\mathrm{pu}}\right.$ $\left.+\omega_{\mathrm{pr}}=16.8 \mathrm{eV}\right)$. The upper panel shows the classical photoelectron kinetic energy expected $\left(\bar{\varepsilon}_{k}=\omega_{\mathrm{pr}}-\Delta V\right.$, where $\Delta V$ is the difference between the ion and neutral state) as a function of geometry $\left(r_{1}=r_{2}=1.22 \AA\right)$ for each ionization channel.

mental wavepacket dynamics in different scenarios: vibrational motion across a one-dimensional double-well potential in an excited state of $\mathrm{Na}_{2},{ }^{24-28}$ wavepacket bifurcation at an avoided crossing in $\mathrm{NaI},{ }^{29,30}$ and proton transfer in the ground state of chloromalonaldehyde. ${ }^{31-33}$

The scheme for our studies of the pump-probe photoelectron spectra in $\mathrm{NO}_{2}$ is illustrated in Fig. $1 . \mathrm{NO}_{2}$ molecules in the ground vibrational level are first transiently aligned using short laser pulses. ${ }^{11,34}$ This initial state is then pumped to an excited electronic state by a femtosecond pulse. Because of the ultrafast time scale of the associated dynamics, we employ pulses of a full-width at halfmaximum (FWHM) of $8 \mathrm{fs}$ in these studies. Wavepacket motion on the excited state, as well as on the ground electronic state that is coupled to the excited state by the CI, is probed with a time-delayed femtosecond pulse that directly ionizes the molecule. The photoelectrons are then energy- and angleresolved for signatures of the wavepacket motion. To simulate these photoelectron spectra, we numerically time-evolve the wavepackets on the relevant electronic surfaces in all three dimensions (neglecting rotation), employing the diabatic representation to handle the nonadiabatic interaction at the CI. The coupling of the electronic surfaces due to the pump and probe pulses is explicitly included in the Hamiltonian, and geometry- and energy-dependent photoionization matrix elements are employed throughout.
The photoelectron spectrum of $\mathrm{NO}_{2}$ has been well characterized. ${ }^{35-37}$ The ground state of the cation is linear, and ionization from the bent neutral ground state results in a long vibrational progression. The first excited state of the ion, ${ }^{3} A^{\prime}$ (or ${ }^{3} B_{2}$ in $C_{2 v}$ notation), is bent at its equilibrium geometry. ${ }^{38,39}$ We consider this state to be a better candidate for probing the dynamics at the CI because of the relative proximity of its equilibrium geometry to the geometry of the CI. Although there are several excited states of the ion lying close in energy to this state at the geometry of the CI, ${ }^{38}$ most of these are dissociative ${ }^{35}$ and we expect photoelectrons associated with this ion surface to be discernible from others. In our previous study ${ }^{23}$ employing model (constant) photoionization amplitudes, the time-resolved photoelectron energy spectra to the triplet state were seen to track the vibrational wavepacket through the CI. The singlet surface of the ion lies close in energy to the triplet state at the geometry of the CI (Ref. 38), and ionization to the singlet state can be expected to make it more difficult to unravel the photoelectron signal from the wavepackets.

The remainder of this paper is organized as follows. Section II outlines the theoretical formulation used in this work, emphasizing the incorporation of ab initio photoionization matrix elements into the description of the photoelectron spectra of the wavepackets. Computational methods and details are discussed in Sec. III, and the time-dependent photoelectron kinetic energy spectra and angular distributions are discussed in Sec. IV. Section V concludes the paper.

\section{FORMULATION}

Our formulation of time-resolved pump-probe photoelectron spectroscopy is fully discussed in earlier papers, ${ }^{25,30}$ and here we present just a brief outline with emphasis on the use of geometry- and energy-dependent photoionization matrix elements.

The wave function of the total system, $\Psi(\boldsymbol{r}, \boldsymbol{R}, t)$, is expanded in the electronic wave functions relevant to the pump-probe arrangement,

$$
\psi(\boldsymbol{r}, \boldsymbol{R}, t)=\sum_{i=X, A} \chi_{i}(\boldsymbol{R}, t) \Phi_{i}(\boldsymbol{r} ; \boldsymbol{R})+\int d \boldsymbol{k} \chi_{k}(\boldsymbol{R}, t) \Phi_{k}^{(-)}(\boldsymbol{r} ; \boldsymbol{R}),
$$

where $i$ labels the adiabatic ground $(X)$ and excited $(A)$ electronic wave functions, $\Phi_{i}(\boldsymbol{r} ; \boldsymbol{R})$, of the neutral molecule and $\Phi_{\boldsymbol{k}}^{(-)}(\boldsymbol{r} ; \boldsymbol{R})$ is the wave function of the final state (ion plus photoelectron). The latter is labeled with the continuous photoelectron wave vector $\boldsymbol{k}, \boldsymbol{R}$ is the set of internal nuclear coordinates, $\boldsymbol{r}$ is the electronic coordinates, and $t$ is the time. Because of the ultrafast time scale of the vibrational dynamics of interest here and also because of the large bandwidth of the ultrashort pulses employed, molecular rotation is neglected in this study. Thus, $\chi_{i}(\boldsymbol{R}, t)$ and $\chi_{\boldsymbol{k}}(\boldsymbol{R}, t)$ are identified with vibrational wave functions in the neutral and ionized systems, respectively.

Coupled equations for the vibrational wave functions can then be written as 


$$
\begin{aligned}
i \frac{\partial}{\partial t}\left(\begin{array}{c}
\chi_{X} \\
\chi_{A}
\end{array}\right)= & {\left[\hat{\mathbf{T}}+\left(\begin{array}{cc}
V_{X}(\boldsymbol{R}) & V_{\mathrm{pu}}(\boldsymbol{R}, t) \\
V_{\mathrm{pu}}(\boldsymbol{R}, t) & V_{A}(\boldsymbol{R})
\end{array}\right)\right]\left(\begin{array}{c}
\chi_{X} \\
\chi_{A}
\end{array}\right) } \\
& +\int d \boldsymbol{k}\left(\begin{array}{c}
V_{\mathrm{pr}}^{(X) *}(\boldsymbol{R}, t) \\
V_{\mathrm{pr}}^{(A) *}(\boldsymbol{R}, t)
\end{array}\right) \chi_{k},
\end{aligned}
$$

and

$$
i \frac{\partial}{\partial t} \chi_{k}=\left[\hat{T}_{\boldsymbol{R}}+V_{\mathrm{ion}}(\boldsymbol{R})+\varepsilon_{k}\right] \chi_{k}+\sum_{i=X, A} V_{\mathrm{pr}}^{(i)} \chi_{i},
$$

where atomic units are used throughout. $\hat{T}_{\boldsymbol{R}}$ is the diagonal kinetic energy operator for the nuclear coordinates, $V_{i}(\boldsymbol{R})$ is the potential energy surfaces of the neutral molecule, $V_{\text {ion }}(\boldsymbol{R})$ is a potential energy surface of the molecular ion, and $\varepsilon_{k}$ is the photoelectron energy (labeled by the photoelectron wave number $k$ ). $\hat{\mathbf{T}}$ is the $2 \times 2$ kinetic energy matrix operator in the adiabatic electronic basis, where off-diagonal terms appear because of the nonadiabatic coupling (CI) between the two electronic states of this system. We resort to quasidiabatization to handle this term in the numerical computation, as explained in the next section. Thus the coupling of the surfaces enters through the potential energy term, and the kinetic energy operator is made diagonal. $V_{\mathrm{pu}}(\boldsymbol{R}, t)$ is the pump-pulse interaction coupling the ground and excited neutral states in the dipole approximation as

$$
V_{\mathrm{pu}}(\boldsymbol{R}, t)=-E_{\mathrm{pu}} f_{\mathrm{pu}}(t) \cos \left(\omega_{\mathrm{pu}} t\right) \mu_{12}(\boldsymbol{R}),
$$

where $E_{\mathrm{pu}}$ is the strength of the pump field, $f_{\mathrm{pu}}$ is the pumppulse envelope, $\omega_{\mathrm{pu}}$ is the pump frequency, and $\mu_{12}(\boldsymbol{R})$ is the transition dipole amplitude along the polarization of the pump pulse. The complex function $V_{\mathrm{pr}}^{(i)}(\boldsymbol{k}, \boldsymbol{R}, t, \Delta T, \hat{\Omega})$ is the probe pulse interaction, to be discussed further shortly. This interaction is by the probed neutral electronic surface, $i$, the delay time from the center of pump pulse, $\Delta T$, and the angles between the probe pulse polarization and the molecular axis, $\hat{\Omega}$.

The electronic wave function of the ion state is written as an antisymmetrized product of an ion wave function, $\Phi_{+}(\boldsymbol{r} ; \boldsymbol{R})$, and a photoelectron orbital, $\phi_{\boldsymbol{k}}^{(-)}(\boldsymbol{r} ; \boldsymbol{R})$,

$$
\Phi_{\boldsymbol{k}}^{(-)}(\boldsymbol{r} ; \boldsymbol{R})=\mathcal{A}\left(\Phi_{+}(\boldsymbol{r} ; \boldsymbol{R}) \cdot \phi_{\boldsymbol{k}}^{(-)}(\boldsymbol{r} ; \boldsymbol{R})\right),
$$

and $\phi_{\boldsymbol{k}}^{(-)}(\boldsymbol{r} ; \boldsymbol{R})$ is expanded in spherical harmonics, $Y_{l \lambda}(\hat{k})$, with $\hat{k}$ being the angular part of $\boldsymbol{k}$,

$$
\phi_{\boldsymbol{k}}^{(-)}(\boldsymbol{r} ; \boldsymbol{R})=\sum_{l \lambda} i^{l} e^{-i \eta_{l}} Y_{l \lambda}^{*}(\hat{k}) \psi_{k l \lambda}^{(-)}(\boldsymbol{r} ; \boldsymbol{R}) .
$$

In Eq. (6) $\boldsymbol{r}$ indicates the electronic coordinates in the molecular frame, $\psi_{k l \lambda}^{(-)}(\boldsymbol{r} ; \boldsymbol{R})$ is a partial wave component of the photoelectron orbital in the molecular frame with momentum $k, \lambda$ is the projection of $l$ in the molecular frame, and $\eta_{l}$ is the Coulomb phase shift. ${ }^{40}$

In the dipole approximation, the probe interaction, $V_{\mathrm{pr}}$, becomes

$$
V_{\mathrm{pr}}=-E_{\mathrm{pr}} f_{\mathrm{pr}}(t-\Delta T) \cos \left(\omega_{\mathrm{pr}} t\right) D,
$$

where the dipole operator, $D$, is

$$
D=\sqrt{\frac{4 \pi}{3}} r \sum_{\mu} \mathcal{D}_{\mu 0}^{1}(\hat{\Omega}) Y_{1 \mu}(\hat{r})
$$

for the linearly polarized case. Here $E_{\mathrm{pr}}$ is the probe field strength, $f_{\mathrm{pr}}(t-\Delta T)$ is the probe pulse envelope, $\omega_{\mathrm{pr}}$ is the probe frequency, $\Delta T$ is the delay time from the center of the pump pulse, $r$ and $\hat{r}$ are the magnitude and angular part of $\boldsymbol{r}$, respectively, and the angles $\hat{\Omega}$ orient the probe polarization with respect to the molecule through the rotation matrix $\left(\mathcal{D}_{\mu 0}^{1}\right)$.

The probe pulse interaction, $V_{\mathrm{pr}}^{(i)}(\boldsymbol{k}, \boldsymbol{R}, t, \Delta T, \hat{\Omega})$, for ionization from the state $i$ then has the form

$$
\begin{aligned}
V_{\mathrm{pr}}^{(i)}(k, \boldsymbol{R}, t, \Delta T, \hat{\Omega})= & \left\langle\Phi_{\boldsymbol{k}}^{(-)}(\boldsymbol{r} ; \boldsymbol{R})\left|V_{\mathrm{pr}}\right| \Phi_{i}(\boldsymbol{r} ; \boldsymbol{R})\right\rangle_{\boldsymbol{r}} \\
= & -E_{\mathrm{pr}} f_{\mathrm{pr}}(t-\Delta T) \cos \left(\omega_{\mathrm{pr}} t\right) \\
& \times \sum_{l \lambda} C_{l \lambda}^{(i)}(k, \boldsymbol{R}, \hat{\Omega}) Y_{l \lambda}(\hat{k}),
\end{aligned}
$$

where

$$
C_{l \lambda}^{(i)}(k, \boldsymbol{R}, \hat{\Omega})=\sqrt{\frac{4 \pi}{3}} \sum_{\mu} I_{l \lambda \mu}^{(i)}(k, \boldsymbol{R}) \mathcal{D}_{\mu 0}^{1}(\hat{\Omega}),
$$

and the bracket subscript $\boldsymbol{r}$ denotes integration over only the electronic coordinates. $I_{l \lambda \mu}^{(i)}(k, \boldsymbol{R})$ is a partial wave matrix element in the molecular frame. These are formed from dipole matrix elements between $\left|\Phi_{+} \psi_{k l \lambda}^{(-)}\right\rangle$and the components of the wave function $\Phi_{i}$. These $C_{l \lambda}^{(i)}(k, \boldsymbol{R}, \hat{\Omega})$ coefficients provide a geometry- and energy-dependent description of the photoionization process.

The ion vibrational wave function, $\chi_{k}(\boldsymbol{R}, t)$, is also expanded in spherical harmonics as

$$
\chi_{\boldsymbol{k}}(\boldsymbol{R}, t)=\sum_{l \lambda} \chi_{k l \lambda}(\boldsymbol{R}, t) Y_{l \lambda}(\hat{k}),
$$

and integration over $\boldsymbol{k}$ in Eq. (2) becomes an integration over $k$ and summation over $l$ and $\lambda$. Integration over $k$ is handled by a quadrature (with weights $w_{j}$ ) over discrete points $k_{j}(j$ $\left.=1,2, \ldots, N_{k}\right)$, where the integration is terminated at a maximum wave number $k_{N_{k}}$. With discretization of both the wave number and angle, the ion vibrational wave function is represented by a set of wave functions $\left\{\chi_{k_{j} l \lambda}(\boldsymbol{R}, t)\right\}$, each associated with different photoelectron energies and angles. For $N_{l}$ sets of $(l, \lambda)$ included in the calculation, the number of coupled equations of motion is thus $\left(2+N_{k} N_{l}\right)$ for the two neutral states and the discretized final state. Equations (2) and (3) are then discretized and solved numerically (Sec. III C).

After propagation of the vibrational wavepackets for a delay time $\Delta T$, the final ion population, $P_{\text {ion }}(\Delta T)$, can be obtained by integrating over $\boldsymbol{k}$, 


$$
\begin{aligned}
P_{\text {ion }}= & \int d \boldsymbol{k} \int d \boldsymbol{R}\left|\chi_{\boldsymbol{k}}\left(\boldsymbol{R}, t_{\mathrm{f}}\right)\right|^{2} \\
\approx & \sum_{j=1}^{N_{k}} w_{j} k_{j}^{2} \int_{0}^{2 \pi} d \phi_{k} \int_{0}^{\pi} \sin \theta_{k} d \theta_{k} \int d \boldsymbol{R} \\
& \times\left|\sum_{l \lambda} \chi_{k_{j} l \lambda}\left(\boldsymbol{R}, t_{\mathrm{f}}\right) Y_{l \lambda}\left(\theta_{k}, \phi_{k}\right)\right|^{2} \\
\equiv & \sum_{j=1}^{N_{k}} w_{j} \int_{0}^{2 \pi} d \phi_{k} \int_{0}^{\pi} \sin \theta_{k} d \theta_{k} P_{k_{j}}\left(\theta_{k}, \phi_{k}\right) \\
\equiv & \sum_{j=1}^{N_{k}} w_{j} k_{j} P\left(\varepsilon_{k}\right),
\end{aligned}
$$

where $t_{f}$ is the time after the probe pulse interaction is over and $\hat{k}=\left(\theta_{k}, \phi_{k}\right)$. The photoelectron kinetic energy distribution, $P\left(\varepsilon_{k}\right)$, and energy-resolved molecular frame photoelectron angular distribution, $P_{k_{j}}\left(\theta_{k}, \phi_{k}\right)$, are given by the integrands of Eq. (12).

The angular coordinates $\left(\theta_{k}, \phi_{k}\right)$ and the ion partial waves can be transformed to a frame aligned with the probe polarization,

$$
\tilde{\chi}_{l m}=\sum_{\lambda} \mathcal{D}_{m \lambda}^{l}(\hat{\Omega}) \chi_{l \lambda}
$$

where the tilde over variables denote the transformed frame. Integration over the $\widetilde{\phi}$ angle around the polarization axis of the probe results in averaged angular distributions of the form

$$
\begin{aligned}
P_{k_{j}}\left(\tilde{\theta}_{k}\right) & =k_{j}^{2} \int_{0}^{2 \pi} d \widetilde{\phi}_{k} \int d \boldsymbol{R}\left|\sum_{l m} \tilde{\chi}_{k_{j} l m} Y_{l m}\left(\tilde{\theta}_{k}, \widetilde{\phi}_{k}\right)\right|^{2} \\
& =k_{j}^{2} \int d \boldsymbol{R}\left|\sum_{l m} \tilde{\chi}_{k_{j} l m} Y_{l m}\left(\tilde{\theta}_{k}, 0\right)\right|^{2}
\end{aligned}
$$

This averaging results in angular distributions from a set of molecules with their molecular axes aligned along one direction.

\section{COMPUTATION}

\section{A. Potential energy surfaces}

The lowest two ${ }^{2} A^{\prime}$ potential surfaces of $\mathrm{NO}_{2}$ in the present study are the same as those reported in our previous paper. ${ }^{23}$ These were obtained as interpolated surfaces over a dense grid of bond lengths $\left(r_{1}, r_{2}\right)$ and bond angles $(\beta)$ with the state-averaged full-valence complete active space selfconsistent-field method as implemented in the MOLPRO quantum chemistry package, ${ }^{41-43}$ using Dunning's correlation consistent polarized triple zeta basis set. ${ }^{44}$ The neutral ground state has an equilibrium bond length of $1.20 \AA$ and bond angle of $133^{\circ}$ [1.196 $\AA$ and $134.3^{\circ}$ in the multireference configuration interaction (MRCI) calculation of Ref. 20], while the first excited ${ }^{2} A^{\prime}$ state has a minimum at a smaller bond angle of $102^{\circ}$ (101.4 $4^{\circ}$ in Ref. 20). The CI is located near the excited-state minimum, with the cusp, for example, located at bond length of $1.26 \AA$, bond angle of $107.5^{\circ}$, and energy of $1.21 \mathrm{eV}$. These can be compared with the values of $1.248 \AA, 106.6^{\circ}$, and $1.28 \mathrm{eV}$, respectively, in the MRCI calculation in Ref. 20.

The surfaces were diabatized with the phenomenological method of Hirsch et al. ${ }^{17}$ for convenience in propagating the wavepacket through the CI. For every bond length in the symmetric $\left(C_{2 v}\right)$ geometry, the $\mathrm{CI}$ arises at a specific angle. Observing that the diabatic surfaces collectively coincide with the adiabatic surfaces at $C_{2 v}$ geometry and that the dipole moment component perpendicular to the bond angle bisector is zero in $C_{2 v}$ geometry and increases away from that geometry, the magnitude of this dipole moment component is used to characterize the diabatic system. The transformation angle, and hence the transformation matrix, from the adiabatic to diabatic representation is obtained by minimizing the diagonal elements of the dipole moment matrix at each geometry. The nonadiabatic coupling constants for the diabatic representation obtained this way are confirmed to be small by direct calculation at several representative geometries. A comparison of the resulting surfaces with other higher level surfaces in the literature ${ }^{19,20}$ showed them to be adequate for describing the dynamics around the CI. Hereafter the neutral states are referred to as state $1\left({ }^{2} A_{1}\right)$ and state $2\left({ }^{2} B_{2}\right.$; see Fig. 1$)$ in the diabatic representation.

There have been far fewer studies of the global surfaces for the $\mathrm{NO}_{2}^{+1} A^{\prime}$ ground state $\left({ }^{1} \Sigma_{\mathrm{g}}^{+}\right.$at the equilibrium geometry) and for the ${ }^{3} A^{\prime}$ triplet ground state $\left({ }^{3} B_{2}\right.$ at $C_{2 v}$ geometry $)^{38,39}$ than for the neutral system. The ion surfaces used in the present study were obtained with the same method and basis set as the neutral surfaces. However, each surface was computed separately without any stateaveraging. The resulting singlet and triplet ion surfaces were shifted vertically up by 1.9 and $1.7 \mathrm{eV}$, respectively, to bring them into agreement with the experiment. ${ }^{36}$ The singlet ground state of the ion has its minimum at a linear geometry and a rather steep slope in the Franck-Condon region for ionization from the bottom of the neutral ground state (see Fig. 1). The first excited state $\left({ }^{3} B_{2}\right.$ in $C_{2 v}$ geometry) is bent and has an equilibrium geometry much closer to the CI region of interest. In the same region the two surfaces approach each other and are both between 13 and $14 \mathrm{eV}$ from the bottom of the neutral state 1 (Fig. 1).

The four ionization channels for the coupled neutral states included in the present studies are shown in the lower panel of Fig. 1 by vertical arrows. In $C_{2 v}$ geometry the dominant configurations of the diabatic neutral states 1 and 2 are $\cdots\left(4 b_{2}\right)^{2}\left(6 a_{1}\right)^{1}$ and $\cdots\left(4 b_{2}\right)^{1}\left(6 a_{1}\right)^{2}$, respectively. Ionization from either the $4 b_{2}$ orbital of state 1 or the $6 a_{1}$ orbital of state 2 leads to the triplet ion. We hereafter refer to these channels as $1 \mathrm{~T}$ (ionization of state 1 to the triplet ion) and $2 \mathrm{~T}$ (ionization of state 2 to the triplet ion). The singlet ion state has a dominant configuration of $\cdots\left(4 b_{2}\right)^{2}\left(6 a_{1}\right)^{0}$ for bond angles larger than $100^{\circ}$, which changes to $\cdots\left(4 b_{2}\right)^{0}\left(6 a_{1}\right)^{2}$ for smaller bond angles. Therefore for bond angles larger than $100^{\circ}$, only the neutral state 1 can ionize to the singlet ion, and we refer to this channel as $1 \mathrm{~S}$. For bond angles less than $100^{\circ}$, only the neutral state 2 can ionize to the singlet ion, and we refer to this channel as $2 \mathrm{~S}$. Note that for ionization to the triplet ion, the $1 \mathrm{~T}$ and $2 \mathrm{~T}$ channels are open for all bond angles, in contrast to the case of ionization to the singlet ion. 
The upper panel of Fig. 1 shows the photoelectron kinetic energy expected classically in the Condon approximation,

$$
\begin{aligned}
\bar{\varepsilon}_{k}(\boldsymbol{R}) & =\left(E_{\mathrm{pr}}-V_{\mathrm{ion}, j}(\boldsymbol{R})\right)-\left(E_{\mathrm{pu}}-V_{i}(\boldsymbol{R})\right) \\
& =\omega_{\mathrm{pr}}-\left(V_{\mathrm{ion}, j}(\boldsymbol{R})-V_{i}(\boldsymbol{R})\right),
\end{aligned}
$$

where $E_{\mathrm{pu}}=v_{0}+\omega_{\mathrm{pu}}$ and $E_{\mathrm{pr}}=v_{0}+\omega_{\mathrm{pu}}+\omega_{\mathrm{pr}}$ are the energies reached by the pump and probe pulses, respectively, $v_{0}$ is the zero point energy, and the indices $i=1,2$ and $j=\mathrm{S}, \mathrm{T}$ refer to the neutral state probed and the final ion state, respectively. The first passage of the wavepacket through the CI is nearly one-dimensional along the bond angle coordinate but with a small amount of symmetric stretch, so a one-dimensional plot as in Fig. 1 is useful. This figure shows the photoelectron kinetic energies only in the regions where each ionization channel is open. The open ionization channels change with bond angles, and each channel produces different photoelectron energies. Changes in the ionization channels with bond angle can be used to monitor the evolution of the wavepacket on the potential surfaces.

\section{B. Photoionization matrix elements}

The procedure for obtaining the photoionization matrix elements has been discussed elsewhere, ${ }^{40}$ and here we give only a brief summary. For the final state wave functions (ion plus photoelectron), we assume a frozen-core Hartree-Fock model in which the ion orbitals are taken to be those of the neutral core and the photoelectron orbital is obtained as a solution of a one-electron Schrödinger equation containing the Hartree-Fock potential of the molecular ion, $V_{\text {ion }}(\boldsymbol{r} ; \boldsymbol{R})$,

$$
\left(-\frac{1}{2} \nabla^{2}+V_{\text {ion }}(\boldsymbol{r} ; \boldsymbol{R})-\varepsilon_{k}\right) \psi_{k l \lambda}^{(-)}(\boldsymbol{r} ; \boldsymbol{R})=0 .
$$

To obtain the partial wave photoelectron orbitals $\psi_{k l \lambda}^{(-)}$, we use an iterative procedure, based on the Schwinger variational principle, to solve the Lippmann-Schwinger equation associated with Eq. (16). ${ }^{40}$ The procedure begins by approximating the static-exchange potential of the relaxed ion core by a separable form,

$$
U_{\mathrm{SE}} \approx U_{\mathrm{S}}\left(\boldsymbol{r}, \boldsymbol{r}^{\prime}\right)=\sum_{i j}\left\langle\boldsymbol{r}|U| \alpha_{i}\right\rangle\left(U^{-1}\right)_{i j}\left\langle\alpha_{j}|U| \boldsymbol{r}^{\prime}\right\rangle,
$$

where the matrix $U^{-1}$ is the inverse of the matrix with the elements $(U)_{i j}=\left\langle\alpha_{i}|U| \alpha_{j}\right\rangle$, the $\alpha$ 's are discrete basis functions such as Cartesian or spherical Gaussian functions, and $U$ is twice the static-exchange potential in Eq. (16) with the longrange Coulomb potential removed. The LippmannSchwinger equation with this separable potential $U_{\mathrm{S}}\left(\boldsymbol{r}, \boldsymbol{r}^{\prime}\right)$ can be readily solved and provides an approximate photoelectron orbital $\psi_{k l \lambda}^{(0)}$. These solutions can be iteratively improved to yield converged solutions to the LippmannSchwinger equation containing the exact static-exchange potential $U_{\mathrm{SE}}$. Several iterations usually provide converged solutions of Eq. (16).

\section{Propagation of the vibrational wave functions}

Details of the procedure for time propagation were given in a previous paper, ${ }^{23}$ and we only briefly outline the method here. The equations of motion for the vibrational wave functions, Eqs. (2) and (3), were cast in discretized matrix form in Jacobi coordinates, $(r, R, \theta)$, where $r$ denotes the distance between $\mathrm{N}$ and one of the $\mathrm{O}$ atoms and the distance $R$ and angle $\theta$ define the vector from the center-of-mass of the NO moiety to the other $\mathrm{O}$ atom. The equations were solved numerically with a split-operator short-time propagator method. More specifically, the time-propagation operator is split into five terms: ${ }^{30}$ the kinetic energy, the diagonal potential, potential coupling between the neutral states due to the $\mathrm{CI}$ and also by the pump pulse, ionization out of the neutral state 1 , and ionization out of state 2 .

The kinetic energy term is handled in the diabatic representation ${ }^{45}$ employing the usual fast Fourier transform (FFT) grid method ${ }^{46}$ for the length coordinates and an FFT grid method devised by Dateo and Metiu for the angle coordinate. ${ }^{47}$ To focus on the early wavepacket dynamics near the CI, the range of the grid was reduced from that of our previous paper ${ }^{23}$ to 143 points between $80^{\circ}$ and $180^{\circ}$ for the $\theta$ coordinate, 54 points between 1.15 and $2.33 \AA$ for $R$, and 56 points between 0.90 and $2.34 \AA$ for $r$. For the early times considered in this paper, dissociation is not significant. The grid parameters were chosen to enable representation of up to $4.0 \mathrm{eV}$ in kinetic energy.

The off-diagonal potential energy terms are diagonalized at each short-time step. Because of the large number of ion partial wave functions, diagonalizing a very large matrix for every spatial grid point is necessary for time propagation. Though this may seem prohibitive, the split form of the ionization part of the time-propagation operator is actually a sparse matrix with nonzero elements only in a single row and a single column, which can be diagonalized in a time linear with the size of the matrix. This case is much more efficient than the general case.

The molecule is taken to lie in the $x y$-plane, and for $C_{2 v}$ geometry, the two $\mathrm{O}$ atoms lie parallel to the $x$-axis, and the $\mathrm{N}$ atom lies on the negative $y$ axis. The pump pulse is polarized along the $x$-axis and the polarization of the probe is taken parallel to that of the pump. We further assume that the molecules are initially transiently aligned using short laser pulses. ${ }^{11,34}$ The propagation of the vibrational wave function is done entirely in internal (Jacobi) coordinates, and the molecular orientation enters into the calculation only through the matrix elements of the pump and probe couplings. The system is propagated from the lowest vibrational level of the ground electronic state with a time step $\Delta t$ of $0.1 \mathrm{fs}$. The excited-state wavepacket is generated with a pump pulse of $\omega_{\mathrm{pu}}=3.1 \mathrm{eV}$ and a Gaussian envelope with a FWHM of $8 \mathrm{fs}$. The center of the pump pulse is taken as time $t=0$ fs. Photoelectron spectra of the resulting wavepackets were then obtained for various delay times, $\Delta T$, for a probe pulse with $\omega_{\mathrm{pr}}=13.5 \mathrm{eV}$ and a FWHM of 8 fs. Photoelectron spectra were obtained separately for ionization to the singlet and triplet ion states.

The Hamiltonian matrix for the ionization run includes coupling between either diabatic neutral state and all ion partial wave functions $\left\{\chi_{k_{i} l}\right\}$. The number of quadrature points for the photoelectron kinetic energy $N_{k}$ was 50 for a maximum kinetic energy of $5.0 \mathrm{eV}$. Partial waves up to 


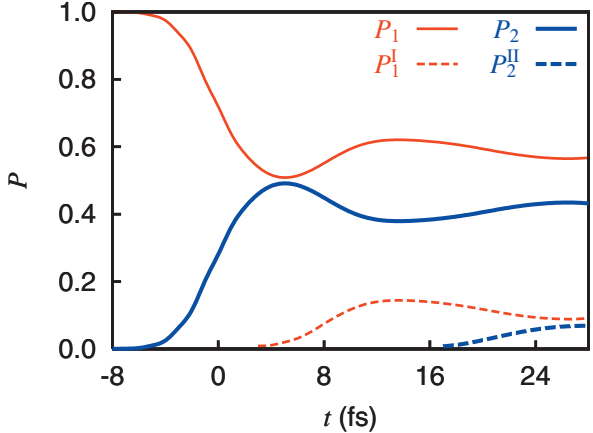

FIG. 2. Time evolution of populations following pump pulse: populations of the two diabatic electronic states $1\left(P_{1}\right)$ and $2\left(P_{2}\right)$ and contributions from the first two passages through the $\mathrm{CI}\left(P_{1}^{\mathrm{I}}\right.$ and $\left.P_{2}^{\mathrm{II}}\right)$ are shown. See text for definitions.

$l=9$ were included in these calculations. An analysis of the resulting spectra shows that waves with $l \leq 5$ accounted for over $99 \%$ of the ion population.

\section{RESULTS AND DISCUSSION}

\section{A. Excited-state wavepacket dynamics}

Figure 2 shows the time evolution of the population of diabatic states 1 and 2 as well as contributions from the first two passages of the wavepacket through the CI. The population of state $1, P_{1}$ (thin red curve), is seen to decrease to $\sim 0.5$ during the pump pulse with a FWHM of 8 fs centered at $t=0 \mathrm{fs}$. Conversely, population builds up in state $2, P_{2}$ (thick blue curve). The wavepacket formed on state 2 by the pump pulse immediately begins to move toward the CI, and the population on state 2 is subsequently affected by the CI. It first reaches the CI by $t=4 \mathrm{fs}$. Most of the population passing through the CI remains on the same diabatic state, but some adiabatically transfer to state 1 , as shown by $P_{1}^{\mathrm{I}}$ (dashed red curve). This component of the population on state 1 is obtained by eliminating the contribution of low vibrational levels with energy $<0.7 \mathrm{eV}$ using calculated vibrational eigenfunctions. The population of the low vibrational levels results from the intense $(I=3.2$ $\times 10^{13} \mathrm{~W} \mathrm{~cm}^{-2}$ ) pump pulse used here, whereas high vibrational levels are populated through the CI.

The first passage through the $\mathrm{CI}$ is complete by around 12 fs. The wavepacket components on the two states then evolve separately, with the component on state 1 returning for a second passage through the CI around $t=20 \mathrm{fs}$, where some of it adiabatically transfer to state 2 (thick blue dashed curve, $\left.P_{2}^{\mathrm{II}}\right)$. The newly formed component on state 2 is spatially well separated from the component on state 2 that remained diabatically on the state during the first crossing of CI for the time span shown, so its population, $P_{2}^{\mathrm{II}}$, can be determined. The increase in $P_{2}^{\mathrm{II}}$ in Fig. 2 near $t=20$ fs indicates the second passage of this component of the wavepacket through the CI.

Figure 3 shows the time evolution of the wavepackets. Each column shows a projection of the amplitude of the wave function onto the $\left(\beta, r_{1}\right)$ plane at times indicated at the top of the panels. The upper row shows the amplitudes of the wavepacket on state 2 , and the lower row the amplitude for

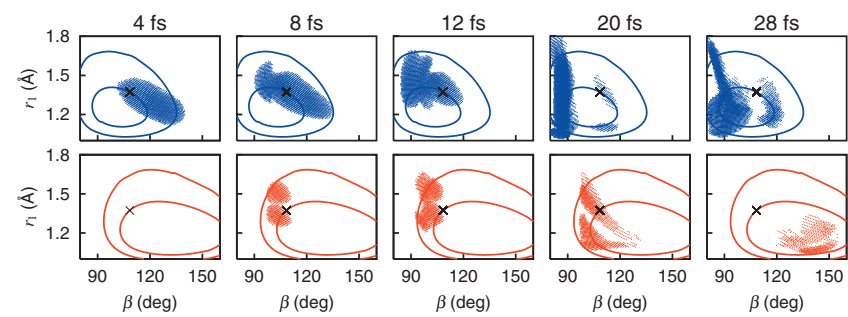

FIG. 3. Time evolution of the wavepacket formed by the pump pulse. Projections of the amplitude of the diabatic wavepacket onto the $\left(\beta, r_{1}\right)$ plane are shown for selected times $t$ indicated at the top. For each column, the upper row shows the amplitude for electronic state 2 and the lower row for state 1. Potential surface contours of the respective electronic states are shown at 3.00 (outer contour) and $1.77 \mathrm{eV}$ (inner), and the $\mathrm{CI}$ is indicated by $\operatorname{cross}(\times)$ for $r_{2}=1.37 \AA$.

state 1. Potential energy contours are shown for $3.00 \mathrm{eV}$ (outer contour) and $1.77 \mathrm{eV}$ (inner contour). The initial wave function left unexcited on state 1 by the pump pulse has been removed for clarity in plotting Fig. 3. A more detailed account of the dynamics and snapshots of wavepackets for later times are given in a previous paper. ${ }^{23}$

\section{B. Time-resolved photoelectron kinetic energy spectra}

Figure 4 shows the time-resolved photoelectron kinetic energy spectra for a probe pulse polarized parallel to the pump polarization ( $x$-axis) and for molecules that have been transiently aligned. The spectra for ionization to the triplet state only are shown in Fig. 4(a). The evolution of the photoelectron spectrum is plotted in steps of $2 \mathrm{fs}$.

A previous study of the time-resolved photoelectron spectra of this system ${ }^{23}$ omitted photoelectrons from the singlet ion channel. That study showed that the wavepacket on the excited state could be tracked by a peak in the photoelectron spectrum initially appearing near $3.2 \mathrm{eV}$ and shifting down to $2 \mathrm{eV}$ as the wavepacket moves toward the CI. This then splits into two components. Ionization of the wavepacket component that passed through the CI results in photoelectrons with energy less than $1.5 \mathrm{eV}$. On the other hand, components that are adiabatically transferred onto state 1 and are moving toward the inner turning point at narrow bond angles result in photoelectrons with energy higher than 1.5 $\mathrm{eV}$. The present photoelectron spectra in Fig. 4(a) show a similar behavior.

Photoelectron kinetic energy spectra for ionization into both the triplet and singlet states are shown in Fig. 4(b). Compared to Fig. 4(a), a broad and intense peak centered at

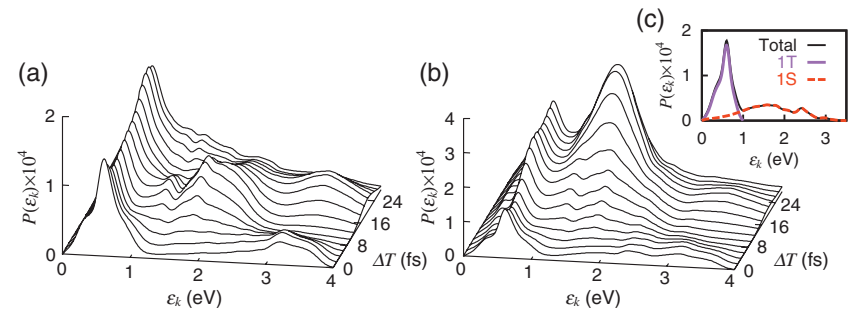

FIG. 4. Time evolution of photoelectron kinetic energy distribution. (a) Ionization to triplet state only. (b) Ionization to both triplet and singlet states. (c) Ionization from the initial wave function only (no pump pulse). 


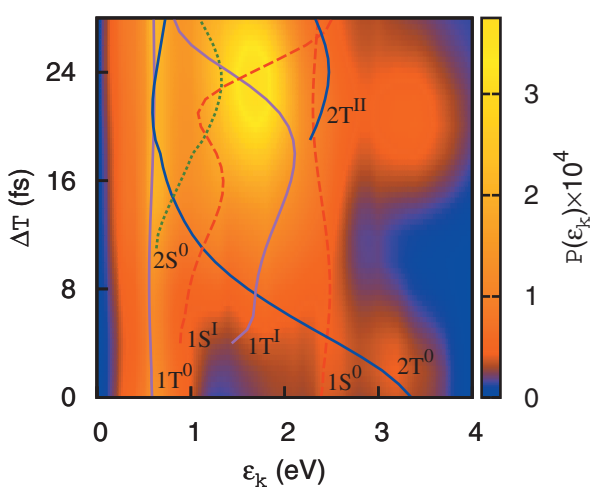

FIG. 5. Chart (top view) of the time-resolved photoelectron kinetic energy spectrum in Fig. 4(b). Curves indicate photoelectron kinetic energies expected for ionization from the centers of the components of the wavepackets. See text for discussion.

$1.7 \mathrm{eV}$ now appears after about 14 fs and obscures the photoelectron spectra from the triplet ion, making it more difficult to infer the wavepacket dynamics from these signals. For comparison Fig. 4(c) shows the photoelectron kinetic energy spectrum for ionization of the initial vibrational eigenstate to the triplet (1T) and singlet (1S) ions in the absence of the pump field.

\section{Interpretation of spectra: Wavepacket components and ionization channels}

The excited wavepacket formed by the pump pulse evolves into several components as seen in Figs. 2 and 3: (1) part of the initial wave function that was not electronically excited by the pump pulse, (2) the bulk of the excited wavepacket formed by the pump pulse, (3) two symmetrically equivalent components formed on electronic state 1 at the time of the first passage through the $\mathrm{CI}\left(P_{1}^{\mathrm{I}}\right.$ in Fig. 2, snapshot shown in the lower panel at 8 fs in Fig. 3), and (4) another set of symmetrically equivalent components formed on electronic state 2 at the time of the second passage through the $\mathrm{CI}\left(P_{2}^{\mathrm{II}}\right.$ in Fig. 2-the additional components shown in the upper panel at 20 fs in Fig. 3). These wavepacket components are probed by the four ionization channels depicted in Fig. 1.

Some insight into these photoelectron energy spectra can be obtained with the help of the classical picture of ionization from the centers of the components of the wavepacket. Figure 5 shows a top view of the same time-resolved photoelectron kinetic energy spectrum as in Fig. 4(b), with the signal strength indicated by color. This chart exposes the qualitative changes in the dynamics more vividly. Curves in this chart show the photoelectron kinetic energy expected at the centers of the various wavepacket components, $\bar{\varepsilon}_{k}\left(\boldsymbol{R}_{c}\right)$, where $\boldsymbol{R}_{c}$ is the expectation value of the coordinate for each wavepacket component. The curves are labeled by ionization channel $(1 \mathrm{~S}, 1 \mathrm{~T}, 2 \mathrm{~S}$, or $2 \mathrm{~T})$ and a superscript indicating the wavepacket component from which ionization occurs (I, the component formed at the first passage through the CI, II, the component formed at the second passage through the CI, and 0 , the bulk of the wave function on state 1 that was electronically unexcited by the pump pulse, or the component on state 2 , formed by the pump pulse but excluding the portions formed at the CI). Triplet ionization channels are shown as solid curves, and singlet ionization channels are shown as dashed (ionization from state 1) or dotted (ionization from state 2) curves. The classical photoelectron energies can be read off a figure such as the upper panel of Fig. 1 using the coordinates of the center of a wavepacket component.

The $1 S^{0}$ (dashed) curve in Fig. 5 represents ionization to the singlet state out of the component of the ground state wavepacket that is electronically unexcited $(\sim 50 \%)$ by the pump pulse and corresponds to the broad $1 \mathrm{~S}$ distribution in Fig. 4(c). The $1 \mathrm{~T}^{0}$ (solid) curve, representing ionization of the same component of the ground state wavepacket to the triplet ion, corresponds to the sharper 1T channel peak in Fig. 4(c). These features do not change with time after the pump pulse is over. The 1T peak in Figs. 4(a) and 4(b) is the dominant feature below $1 \mathrm{eV}$ but lies well below features arising from ionization of excited wavepackets. In contrast, the broad 1S photoelectron distribution obscures features in the time-resolved spectrum above $1 \mathrm{eV}$, making it more difficult to infer excited-state wavepacket dynamics.

As it moves toward its first passage through the CI, the wavepacket formed on state 2 by the pump pulse can be tracked by the $2 \mathrm{~T}$ ionization channel $\left(2 \mathrm{~T}^{0}\right.$, blue curve in Fig. 5), while after passage through the CI, the bulk of the wavepacket can be tracked by the $2 \mathrm{~S}$ channel signal $\left(2 \mathrm{~S}^{0}\right.$, green dotted curve). The $2 \mathrm{~S}$ channel becomes active only after the wavepacket has passed through the $\mathrm{CI}$ and is enhanced as it approaches the turning point in the bending coordinate. The $2 \mathrm{~T}$ channel signal is evident in Fig. 4(a) as a peak above 3 $\mathrm{eV}$ for delay times up to $8 \mathrm{fs}$ and another near $1 \mathrm{eV}$ between 6 and 16 fs. The pronounced peak below $2 \mathrm{eV}$ in Fig. 4(b) after $\Delta T=14$ fs is due to the $2 \mathrm{~S}$ signal. Though the population probed by the $2 \mathrm{~S}$ channel is smaller than that initially probed by the $2 \mathrm{~T}$ channel due to some population loss near the CI, the $2 \mathrm{~S}$ signal is much stronger because of the large photoelectron matrix elements for singlet ionization.

$P_{1}^{\mathrm{I}}$ in Fig. 2 is the population on state 1 due to the first passage of the wavepacket through the CI. This wavepacket component is formed symmetrically on either side of the CI $\left(r_{1}>r_{2}\right.$ or $r_{1}<r_{2}$; see Fig. 3 at 8 fs). Only one of these two components is used to obtain the expectation value of the wavepacket coordinates. Once formed, this wavepacket component can be probed by either the $1 \mathrm{~T}$ channel $\left(1 \mathrm{~T}^{\mathrm{I}}\right.$ curve in Fig. 5) or the $1 \mathrm{~S}$ channel ( $1 \mathrm{~S}^{\mathrm{I}}$ dashed curve). The $1 \mathrm{~S}$ channel signal is too weak to be seen due to the background signal from the unexcited portion of the ground state wavepacket. The 1T signal from this wavepacket, seen as the peak near 2 $\mathrm{eV}$ in Fig. 4(a) between $\Delta T=6$ and $20 \mathrm{fs}$, is, however, obscured by the large $2 \mathrm{~S}$ signal when ionization to the singlet ion is included [Fig. 4(b)].

The curve $P_{2}^{\mathrm{II}}$ in Fig. 2 represents the population of the wavepacket formed on state 2 from the second passage through the CI. While ionization of this wavepacket to the singlet ion is not allowed, ionization to the triplet ion is, and the corresponding classical photoelectron energy is shown by the curve $2 \mathrm{~T}^{\mathrm{II}}$ in Fig. 5. Only one of the two symmetrically formed components is used in obtaining the average coordinate, as in the case for $1 \mathrm{~T}^{\mathrm{I}}$. Though this component can be tracked by the $2 \mathrm{~T}$ signal in principle, the signal, expected 


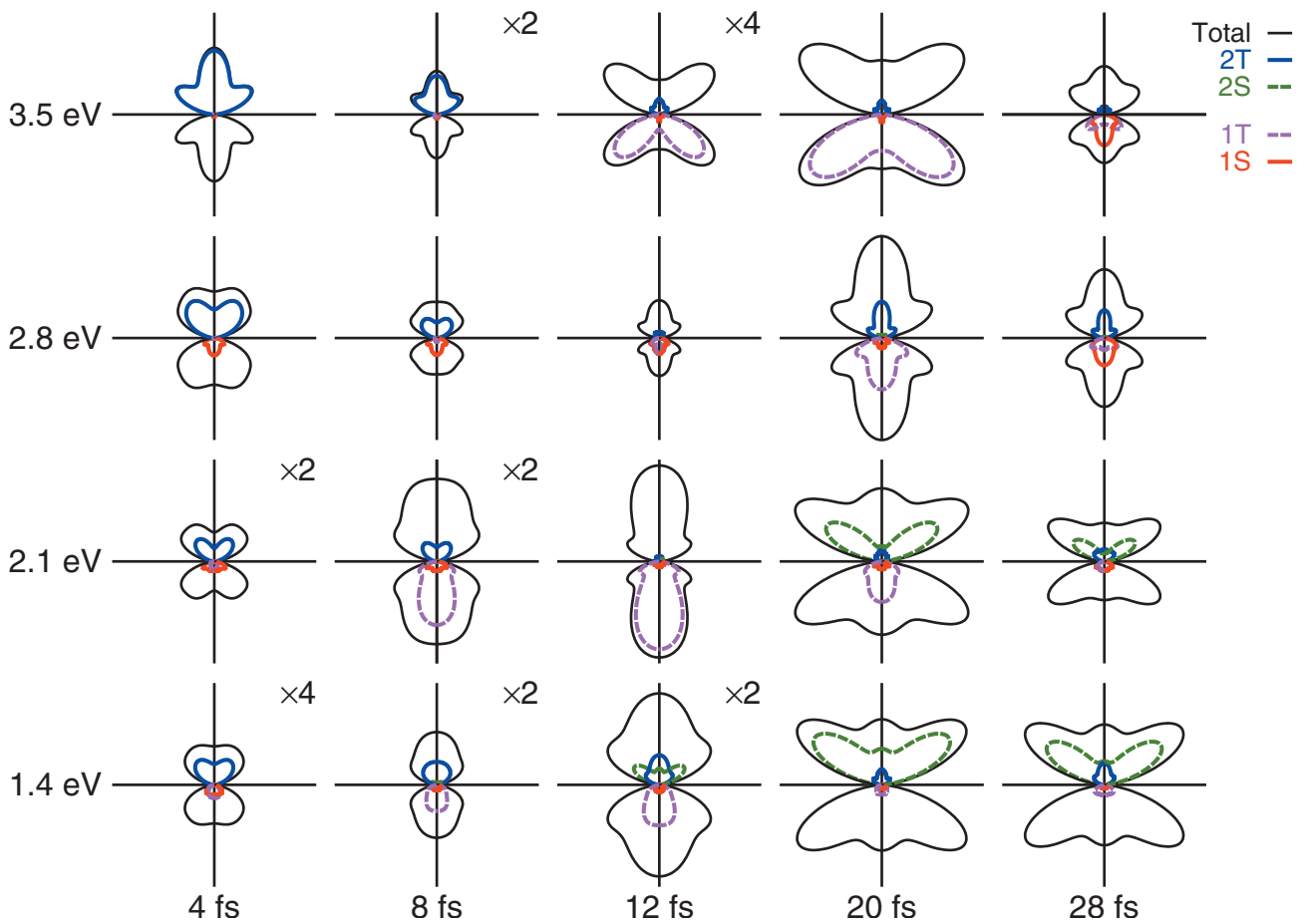

FIG. 6. Evolution of the energy-resolved photoelectron angular distributions. Each row shows the photoelectron angular distributions at various delay times $\Delta T$ for a given photoelectron energy $\varepsilon_{k}$. The upper half of each figure shows the angular distributions associated with the $2 \mathrm{~T}$ (blue curve) and $2 \mathrm{~S}$ (green) ionization channels and the lower half the distributions for the 1T (pink) and 1S (red) channels. The outer contours (black) are the angular distributions summed over all four channels. The pump and probe polarizations lie along the vertical axis, and the angular distributions are rotationally symmetric with respect to this axis. Though only the upper or lower half is shown for each ionization channel, all distributions are symmetric with respect to the horizontal axis. Signal strengths are normalized for each photoelectron kinetic energy separately, and a factor is indicated at the top right when a panel for a particular delay time has been magnified for clearer presentation.

around $\varepsilon_{k}=3.4 \mathrm{eV}$, is too weak to be seen in Fig. 4. Instead, the peak seen around this energy at $\Delta T=22$ fs actually arises from ionization to the triplet ion (1T) of the wavepacket component near the turning point on state 1 . The lower panel of Fig. 3 shows that around $t=20$ fs, the leading edge of the wavepacket on state 1 is at a longer bond length, while the center of the wavepacket is at shorter bond length and moving toward larger bond angles. The average value of the coordinate of the wavepacket for this delay time cannot fully describe its multidimensional character. Although, in principle, the time-resolved photoelectron spectra of the wavepackets on the potential surfaces should make it possible to monitor their associated dynamics, broad overlapping peaks in the calculated photoelectron spectra make such an analysis here difficult for short pump and probe pulses.

\section{Time-resolved photoelectron angular distributions}

While overlapping signals from different ionization channels make it difficult to infer the wavepacket dynamics from the photoelectron kinetic energy spectra in Secs. IV B and IV C, the angular distributions provide a clearer and more useful picture of the underlying ionization channels and wavepacket dynamics around the CI. Figure 6 shows our calculated time- and energy-resolved photoelectron angular distributions from spatially aligned molecules $\left[P_{k}\left(\tilde{\theta}_{k}\right)\right.$ in Eq. (14)] for the same pump and probe pulse parameters as in the photoelectron kinetic energy spectra in Sec. IV B. Each row shows, from left to right, the time evolution of the angular distributions for the photoelectron kinetic energy, $\varepsilon_{k}$, indi- cated on the left. The delay time, $\Delta T$, for each column is shown on the bottom. The probe polarization lies along the vertical axis of each figure and the distributions are symmetric with respect to rotation around this axis as a result of averaging in the laboratory frame [Eq. (14)]. Each figure shows the angular distributions from the individual channels, while the outer contour for each plot shows the angular distribution summed over all four ionization channels. The upper half of each figure shows the angular distributions associated with the $2 \mathrm{~T}$ (blue curve) and $2 \mathrm{~S}$ (green) ionization channels, and the lower half the distributions for the $1 \mathrm{~T}$ (pink) and 1S (red) channels. All distributions are symmetric with respect to the horizontal (molecular) axis, reflecting the overall symmetry of the molecule, i.e., bond lengths $r_{1}>r_{2}$ and $r_{1}<r_{2}$ for the same pair of $\left(r_{1}, r_{2}\right)$ occur with equal probability. Only the upper or lower half of the distributions is drawn for individual components. For clarity the plots are normalized separately for each photoelectron kinetic energy, and hence they do not represent relative strengths of the signal at different energies. Relative strengths of the total signal can be seen in Fig. 4. For a given energy the plots in Fig. 6 show the time evolution of the strength of the signal as well as its shape. For delay times with a weak signal, the plots are magnified by factors indicated at the top right. The contribution of each component is shown to scale with the total signal to illustrate the relative importance of each channel.

These time- and energy-resolved laboratory-frame angular distributions are seen to change with delay time and photoelectron kinetic energy, reflecting the dependence of the 
photoionization amplitudes on geometry and energy. The photoelectron angular distributions for each ionization channel could be used to track the transfer of population among electronic states as the vibrational wavepacket moves through the nonadiabatic region. The signal for $\varepsilon_{k}=3.5 \mathrm{eV}$ illustrates this well. As discussed in Sec. IV B, the wavepacket formed by the pump pulse on state 2 is at first probed through the $2 \mathrm{~T}$ ionization channel ( $3.5 \mathrm{eV}$ and $4 \mathrm{fs}$ in Fig. 6). As this wavepacket goes through the $\mathrm{CI}$ region at $8 \mathrm{fs}$, a part of the wavepacket is adiabatically transferred to state 1 , leading to a signal in the $1 \mathrm{~T}$ ionization channel $(3.5 \mathrm{eV}$ and $12 \mathrm{fs}$ in Fig. 6). After passage through the CI region, the photoelectron energy expected classically in the $2 \mathrm{~T}$ channel is less than $1.5 \mathrm{eV}$ (see upper panel of Fig. 1), and the signal at 3.5 $\mathrm{eV}$ is hence much weaker than the $1 \mathrm{~T}$ signal at this time. This results in a significant change in the overall photoelectron angular distribution as the wavepacket passes through the CI region. When this portion of the wavepacket on state 1 passes through the CI ( $\sim 20 \mathrm{fs})$ again, the $1 \mathrm{~S}$ channel becomes dominant, leading to a significant change in the overall angular distribution (3.5 eV and $28 \mathrm{fs}$ in Fig. 6). These changes in the overall photoelectron angular distributions should make it possible to track the evolution of the wavepacket as population is transferred from one region to another through nonadiabatic interactions.

At $1.4 \mathrm{eV}$ the evolution of the aggregate photoelectron angular distributions in Fig. 6 reflects changes in the distributions within a given channel in addition to changes in active channels. Whereas the angular distributions for each ionization channel have similar shapes throughout at $\varepsilon_{k}$ $=3.5 \mathrm{eV}$, at $1.4 \mathrm{eV}$ the distributions for the $2 \mathrm{~T}$ channel (between 4 and $12 \mathrm{fs}$ ) and 1T channel (between 12 and $28 \mathrm{fs}$ ) are seen to change shape (Fig. 6). These changes arise from the geometry dependence of the $C_{l \lambda}$ matrix elements in Eq. (10), with the relative contribution of $(l, \lambda)$ partial waves changing on either side of the CI. The evolution of the angular distributions in the $2 \mathrm{~T}$ channel between 4 and $12 \mathrm{fs}$ at $1.4 \mathrm{eV}$ reflects the passage of the wavepacket through the CI. Removal of the $2 \mathrm{~T}$ contribution would result in aggregate distributions of similar shapes over this time interval. During this time the shape of the angular distributions in the 1T channel remains about the same. These angular distributions in the 1T channel probe the component of the wavepacket formed adiabatically by transfer from diabatic state 2 to state 1 near the CI, and because this component lies only on one side of the CI, no large dependence on geometry is expected. During the second passage of the wavepacket through the CI (12 to $28 \mathrm{fs}$ ), the $1 \mathrm{~T}$ signal tracks the diabatic state 1 population through the $\mathrm{CI}$, and its angular distribution shows a strong dependence on geometry. At the same time, however, there is a strong $2 \mathrm{~S}$ channel signal due to the arrival of wavepacket population from the first CI passage to the small bond angle turning point. This obscures the 1T signal from the second passage through the CI. Nevertheless, these results suggest that evolution of the energy-resolved angular distribution with time even when only one ionization channel is effective can be useful in tracking the wavepacket through a CI. This is in contrast to the angular distributions at $3.5 \mathrm{eV}$,
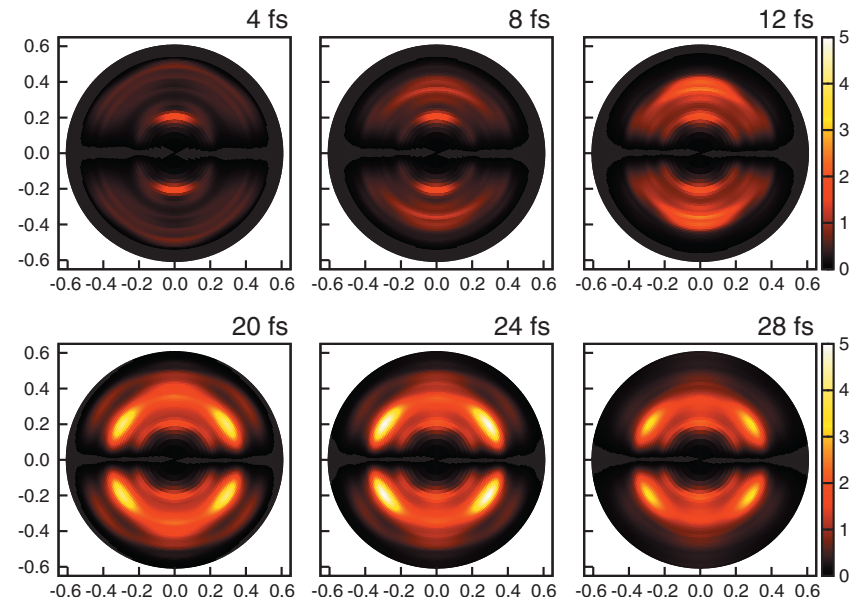

FIG. 7. Femtosecond time-resolved photoelectron images. The vertical axis is the photoelectron momentum along the polarization axis of pump and probe in atomic units.

which essentially monitor the buildup of population due to nonadiabatic interactions in each region probed by different ionization channels.

The evolution of the aggregate photoelectron angular distributions (4-12 fs) at 2.1 and $2.8 \mathrm{eV}$ in Fig. 6 is seen to track the wavepacket as it moves through the CI. Though this behavior is similar to that seen at $3.5 \mathrm{eV}$, it is important to note the significant dependence of these distributions on energy, e.g., $2.8 \mathrm{eV}$ versus $3.5 \mathrm{eV}$. The angular distributions at 2.1 and $2.8 \mathrm{eV}$ also show a dependence on geometry similar to those at $1.4 \mathrm{eV}$, with changes in the $2 \mathrm{~T}$ channel reflecting the first passage of the wavepacket through the CI and changes in the 1T channel its second passage. Changes in the shape of these distributions in the $2 \mathrm{~T}$ channel at $2.1 \mathrm{eV}$, however, occur later than at $1.4 \mathrm{eV}$, while the changes in the distributions at $2.8 \mathrm{eV}$ occur even later. Figure 5 shows the photoelectron kinetic energy expected from a classical view. The wavepacket is composed of a distribution of components with some higher and others lower in nuclear kinetic energy than the average energy. Wavepacket components with higher nuclear kinetic energy should give rise to lowerenergy photoelectrons and vice versa for lower-energy wavepacket components. Hence, around the CI where the angular distributions depend strongly on energy, the dynamics at a lower photoelectron energy should reflect the faster components of the wavepacket, while the higher-photoelectron energy signal reflects the dynamics of the slower part of the wavepacket. Ionization giving rise to $3.5 \mathrm{eV}$ photoelectrons probes a region too far away from the CI compared to the energy spread of the wavepacket for this effect of CI to be observed. Likewise, the $2 \mathrm{~S}$ channel angular distributions, away from nonadiabatic regions, do not change its shape significantly over time.

\section{E. Velocity map imaging}

From the time- and energy-resolved photoelectron angular distributions of the previous section, we can construct femtosecond time-resolved photoelectron images of these spectra, which are shown in Fig. 7. The vertical axis indicates the photoelectron momentum along the direction of the 
pump and probe polarizations in atomic units, and the horizontal axis the momentum perpendicular to it. The intensities $\left[P_{k}\left(\tilde{\theta}_{k}\right) \times 10^{4}\right.$ in Eq. (14)] are indicated by color and brightness. These images offer a compact representation of the angular distribution plots in Fig. 6 over the entire range of photoelectron energies and reaffirm how these distributions track the wavepacket dynamics. The bright innermost ring at $k=0.2$ at early times, mostly in direction parallel to the probe pulse, corresponds to the 1T peak in Fig. 4(c) (photoionization from the unexcited initial state). The $k=0.3$ and $k=0.4$ rings correspond to the 1.4 and $2.1 \mathrm{eV}$ plots in Fig. 6, and we again see the buildup in intensity in the direction parallel to the probe as the wavepacket passes through the CI between 4 and $12 \mathrm{fs}$. At later times (20-28 fs), the peak intensity lies away from the probe polarization and, as shown, comes from the $2 \mathrm{~S}$ and $1 \mathrm{~T}$ channels in Fig. 6. For the outermost ring $(k=0.5)$, the intensity along the diagonal direction from the $1 \mathrm{~T}$ channel weakens relative to the $1 \mathrm{~S}$ channel signal in the parallel direction. This shift indicates the second passage of the wavepacket through the CI, as discussed for the $\varepsilon_{k}=3.5 \mathrm{eV}$ case in Fig. 6 .

\section{CONCLUSIONS}

We have explored the application of femtosecond timeresolved photoelectron spectroscopy for real-time monitoring of wavepacket dynamics through the CI between the first two ${ }^{2} A^{\prime}$ states of $\mathrm{NO}_{2}$. Global potential energy surfaces for the ground and first excited ${ }^{2} A^{\prime}$ states and for the ground singlet and first triplet ion states are employed to time-propagate the quantum vibrational wave function in full (three) dimensions using a short-time propagator. The ab initio geometry- and energy-dependent photoionization amplitudes are explicitly incorporated in these studies.

As in our previous model study, ${ }^{23}$ the time-resolved photoelectron kinetic energy spectra are seen to track the vibrational wavepacket dynamics near the CI. However, overlapping signals from several ionization channels make it difficult to infer the wavepacket dynamics from the photoelectron energy spectra alone. Nevertheless, the evolution of the photoelectron angular distributions make it possible to track the evolution of the wavepackets as population is transferred in nonadiabatic regions. The results suggest that this behavior might arise quite generally in polyatomic molecules.

\section{ACKNOWLEDGMENTS}

This work was supported in part by a Grant-in-Aid for Basic Science from the Ministry of Education, Culture, Sports, Science and Technology of Japan. These studies made use of the resources of the Jet Propulsion Laboratory's Supercomputing and Visualization Facility.

${ }^{1}$ I. V. Hertel and W. Radloff, Rep. Prog. Phys. 69, 1897 (2006).

${ }^{2}$ T. Suzuki, Annu. Rev. Phys. Chem. 57, 555 (2006).

${ }^{3}$ M. Wollenhaupt, V. Engel, and T. Baumert, Annu. Rev. Phys. Chem. 56, 25 (2005)

${ }^{4}$ A. Stolow, A. E. Bragg, and D. M. Neumark, Chem. Rev. (Washington, D.C.) $\mathbf{1 0 4}, 1719$ (2004)

${ }^{5}$ H. Satzger, D. Townsend, M. Z. Zgierski, S. Patchkovskii, S. Ullrich, and
A. Stolow, Proc. Natl. Acad. Sci. U.S.A. 103, 10196 (2006).

${ }^{6}$ R. V. Bensasson, E. J. Land, and T. G. Truscott, Excited States and Free Radicals in Biology and Medicine (Oxford University Press, Oxford, 1993), Chap. 5.

${ }^{7}$ C. E. Crespo-Hernández, B. Cohen, P. M. Hare, and B. Kohler, Chem. Rev. (Washington, D.C.) 104, 1977 (2004).

${ }^{8}$ S. Ullrich, T. Schultz, M. Zgierski, and A. Stolow, Phys. Chem. Chem. Phys. 6, 2796 (2004).

${ }^{9}$ M. Seel and W. Domcke, J. Chem. Phys. 95, 7806 (1991).

${ }^{10}$ V. Blanchet, M. Zgierski, T. Seidemann, and A. Stolow, Nature (London) 401, 52 (1999).

${ }^{11}$ C. Z. Bisgaard, O. J. Clarkin, G. Wu, A. M. D. Lee, O. Geßner, C. C. Hayden, and A. Stolow, Science 323, 1464 (2009).

${ }^{12}$ T. Horio, T. Fujii, Y. Suzuki, and T. Suzuki, J. Am. Chem. Soc. 131, 10392 (2009).

${ }^{13}$ A. Delon, R. Jost, and M. Lombardi, J. Chem. Phys. 95, 5701 (1991).

${ }^{14}$ A. Delon and R. Jost, J. Chem. Phys. 110, 4300 (1999).

${ }^{15}$ A. Delon, R. Jost, and M. Jacon, J. Chem. Phys. 114, 331 (2001).

${ }^{16}$ E. Haller, H. Köppel, and L. S. Cederbaum, J. Mol. Spectrosc. 111, 377 (1985).

${ }^{17}$ G. Hirsch, R. J. Buenker, and C. Petrongolo, Mol. Phys. 70, 835 (1990).

${ }^{18}$ U. Manthe and H. Köppel, J. Chem. Phys. 93, 1658 (1990).

${ }^{19}$ S. Mahapatra, H. Köppel, L. S. Cederbaum, P. Stampfuß, and W. Wenzel, Chem. Phys. 259, 211 (2000).

${ }^{20}$ V. Kurkal, P. Fleurat-Lessard, and R. Schinke, J. Chem. Phys. 119, 1489 (2003).

${ }^{21}$ A. T. J. B. Eppink, B. J. Whitaker, E. Gloaguen, B. Soep, A. M. Coroiu, and D. H. Parker, J. Chem. Phys. 121, 7776 (2004).

${ }^{22}$ M. Sanrey and M. Joyeux, J. Chem. Phys. 125, 014304 (2006).

${ }^{23}$ Y. Arasaki and K. Takatsuka, Chem. Phys. 338, 175 (2007).

${ }^{24}$ Y. Arasaki, K. Takatsuka, K. Wang, and V. McKoy, Chem. Phys. Lett. 302, 363 (1999).

${ }^{25}$ Y. Arasaki, K. Takatsuka, K. Wang, and V. McKoy, J. Chem. Phys. 112, $8871(2000)$

${ }^{26}$ K. Takatsuka, Y. Arasaki, K. Wang, and V. McKoy, Faraday Discuss. 115, 1 (2000)

${ }^{27}$ Y. Arasaki, K. Takatsuka, K. Wang, and V. McKoy, J. Electron Spectrosc. Relat. Phenom. 108, 89 (2000).

${ }^{28}$ Y. Arasaki, K. Takatsuka, K. Wang, and V. McKoy, J. Chem. Phys. 114, 7941 (2001)

${ }^{29}$ Y. Arasaki, K. Takatsuka, K. Wang, and V. McKoy, Phys. Rev. Lett. 90, 248303 (2003).

${ }^{30}$ Y. Arasaki, K. Takatsuka, K. Wang, and V. McKoy, J. Chem. Phys. 119, 7913 (2003).

${ }^{31}$ Y. Arasaki, K. Yamazaki, M. T. do N. Varella, and K. Takatsuka, Chem. Phys. 311, 255 (2005).

${ }^{32}$ M. T. do N. Varella Y. Arasaki, H. Ushiyama, V. McKoy, and K. Takatsuka, J. Chem. Phys. 124, 154302 (2006).

${ }^{33}$ M. T. do N. Varella Y. Arasaki, H. Ushiyama, K. Takatsuka, K. Wang, and V. McKoy, J. Chem. Phys. 126, 054303 (2007).

${ }^{34}$ F. Rosca-Pruna and M. J. J. Vrakking, Phys. Rev. Lett. 87, 153902 (2001).

${ }^{35}$ J. H. D. Eland and L. Karlsson, Chem. Phys. 237, 139 (1998).

${ }^{36}$ P. Baltzer, L. Karlsson, B. Wannberg, D. M. P. Holland, M. A. MacDonald, M. A. Hayes, and J. H. D. Eland, Chem. Phys. 237, 451 (1998).

${ }^{37}$ G. K. Jarvis, Y. Song, C. Y. Ng, and E. R. Grant, J. Chem. Phys. 111, 9568 (1999)

${ }^{38}$ D. M. Hirst, J. Chem. Phys. 115, 9320 (2001).

${ }^{39}$ K. Takeshita and N. Shida, J. Chem. Phys. 116, 4482 (2002).

${ }^{40}$ R. R. Lucchese, D. K. Watson, and V. McKoy, Phys. Rev. A 22, 421 (1980); R. R. Lucchese, G. Raseev, and V. McKoy, ibid. 25, 2572 (1982); S. N. Dixit and V. McKoy, J. Chem. Phys. 82, 3546 (1985); R. R. Lucchese, K. Takatsuka, and V. McKoy, Phys. Rep. 131, 147 (1986); K. Wang and V. McKoy, J. Chem. Phys. 95, 4977 (1991); Annu. Rev. Phys. Chem. 46, 275 (1995).

${ }^{41}$ H.-J. Werner and P. J. Knowles, J. Chem. Phys. 82, 5053 (1985).

${ }^{42}$ P. J. Knowles and H.-J. Werner, Chem. Phys. Lett. 115, 259 (1985).

${ }^{43}$ H.-J. Werner, P. J. Knowles, R. Lindh et al., MOLPRO, version 2006.1, A package of $a b$ initio programs, 2006.

${ }^{44}$ T. H. Dunning, J. Chem. Phys. 90, 1007 (1989).

${ }^{45}$ J. Alvarellos and H. Metiu, J. Chem. Phys. 88, 4957 (1988).

${ }^{46}$ R. Kosloff, J. Phys. Chem. 92, 2087 (1988).

${ }^{47}$ C. E. Dateo and H. Metiu, J. Chem. Phys. 95, 7392 (1991). 